\title{
¿Podré acabar el temario?: Las preocupaciones a las que se enfrenta el profesorado novel
}

\section{Will I finish the syllabus? Concerns of novice teachers}

\author{
Lidón MOLINER MIRAVET y Joana ORTÍ PITARCH \\ Universitat Jaume I
}

Recibido: Febrero 2015

Evaluado: Marzo 2015

Aceptado: Abril 2015

\begin{abstract}
Resumen
El término profesorado novel es un tópico que puede ser abordado desde dos perspectivas diferentes, aquellas que tienen en cuenta los años de experiencia docente y las que hacen hincapié en los rasgos que caracterizan a estos profesionales. Dados los cambios que se han sucedido en la educación del siglo XXI es necesario conocer los principales problemas a los que se enfrenta este tipo de profesorado. En este trabajo, se presenta una investigación cuantitativa basada en el método descriptivo de tipo encuesta llevada a cabo con un total de 50 profesores/as (noveles y experimentados) de dos Comunidades Autónomas, la Comunidad Valenciana y Cataluña en el año 2014. El objetivo es doble, por una parte, conocer las preocupaciones del profesorado novel, y por otra, averiguar si existen diferencias entre las percepciones aportadas por los docentes con experiencia y aquellos más noveles. Para ello, se ha utilizado como instrumento el Inventario de Problemas de la Enseñanza, realizándose un análisis de frecuencias relativas de los datos obtenidos para responder al primer objetivo y la te Student para llevar a cabo el análisis comparativo de los tres grupos estudiados. Los resultados indican que los problemas a los que se enfrenta el profesorado novel de esta investigación tienen que ver con cuestiones relacionadas con las dimensiones académicas y organizativas, además de encontrar que, a mayor experiencia docente, menos problemáticas se perciben en las situaciones presentadas. Se sugieren algunas iniciativas de trabajo colaborativo y de aprendizaje entre iguales para poder mitigar el "choque" del profesorado novel con su nueva realidad como docente.
\end{abstract}

Palabras clave: educación secundaria, bachillerato, formación profesional, profesor novel, preocupaciones.

\begin{abstract}
The term novice teacher is a topic that can be approached from two different perspectives, those that take into account the years of teaching experience and the ones that emphasize the traits that characterize these professionals. Given the changes that have occurred in education in the 21st century it is necessary to know the main problems that these teachers face. In this work, we show a quantitative research based on the descriptive method type survey carried out with a
\end{abstract}


total of 50 teachers (novice and experienced) of two autonomous regions, Valencia and Catalonia in the year 2014. The aim is double, on the one hand, to know the concerns of novice teachers, and on the other, to find out whether there are differences between the perceptions provided by experienced and inexperienced teachers. In order to do this, the Problems of Teaching Inventory has been used as an instrument, performing an analysis of relative frequencies of the data obtained to respond to the first objective and the Student ' $t$ ' to carry out the comparative analysis of the three groups. The results indicate that novice teachers participating in this research face problems that have to do with issues related to academic and organization dimensions, apart from finding out that more teaching experience leads to less problematic situations perceived in the situations to which they were exposed. Some initiatives of collaborative work and peer learning are suggested in order to mitigate the "clash" of novice teachers with their new reality as teachers.

Keywords: secondary education, A levels, vocational training, novice teacher, concerns.

Durante los últimos años, el perfil del profesorado ha ido cambiando a la vez que también ha ido evolucionando la sociedad en la que han de desarrollar su actividad docente, la cual les exige nuevas competencias y conocimientos. Además de ello, la función docente es cada vez más compleja, con un mayor número de retos y demandas que complejizan el quehacer del profesorado (Eirín, García y Montero, 2009). Uno de los factores cruciales ha sido la incorporación de las nuevas tecnologías de la información y las nuevas formas de comunicarnos y relacionarnos. Fuentes de información que, están haciendo necesario un replanteamiento de las funciones que tradicionalmente se han venido asignando a las escuelas y a los profesionales que en ella trabajan: los profesores y profesoras (Marcelo, 2001). Teniendo en cuenta esta nueva realidad podemos hablar de que los profesores han protagonizado en nuestras aulas una profunda revolución silenciosa, obligada por los cambios sociales que han exigido a nuestros sistemas educativos elaborar nuevas respuestas para responder ante nuevos problemas sociales, económicos y políticos (Esteve, 2003). Pero, esta situación ¿afecta de la misma manera al profesorado novel o poco experimentado? ¿Son los nuevos docentes nativos digitales? ¿Quiere decir esto que saben aplicar estas nuevas tecnologías en las aulas? Si a este nuevo contexto le añadimos cómo la iniciación a la docencia es motivo de una constante preocupación se hace necesario cuidar a estos profesionales que comienzan su camino laboral e indagar sobre cuáles son las problemáticas a las que se enfrentan, ya que de ellos y ellas, depende la calidad de la educación futura.

\section{El profesorado novel: conceptualización y rasgos}

La investigación sobre el profesorado novel es un tópico muy estudiado hoy en día, pero no existe una única definición del mismo. En la literatura encontramos muchos conceptos para referirse a este término como por ejemplo: principiante, inexperto, joven, nuevo o neófito. Para llevar a cabo una descripción sobre el término, a continuación presentamos, por un lado, varios autores que conceptualizan el profesorado novel en función de los años de experiencia. Y por otra parte, varios 
autores que lo describen según los rasgos que los caracterizan y que se diferencian del resto del profesorado con experiencia docente superior a 4 años. Esta distinción se ha realizado teniendo en cuenta el estudio de Cañón (2012).

No existe un consenso en cuanto a los años de docencia que deben superarse para dejar de ser novel. Tomando como referencia a diversos autores, consideramos que un profesor o profesora es novel desde el primer año de ejercicio (Marcelo, 2009; Breuse et, al, 1984) hasta el cuarto año (Veenman, 1988) dado que al finalizar este año, su figura de profesor/a ya se encuentra perfilada (Cañón, 2012).

En cuanto a los rasgos que caracterizan al profesorado novel destacamos aquellos que hacen referencia a una persona joven que transita desde el aprendiz de conocimientos, habilidades y destrezas hasta el profesional que pone en práctica lo aprendido en un contexto real (Vera, 1988), aquel que enseña y aprende a enseñar (Feiman-Nemser, 2001); el que posee condición de extranjero en un mundo extraño (Sabar, 2004) y a la persona insegura que busca el apoyo de sus compañeros/as más expertos (Johnston y Ryan, 1983; Saint-Onge, 1997). Por tanto, en el campo educativo, el profesorado novel es aquel que se ha graduado recientemente y que empieza a impartir la docencia por primera vez en un centro, y que se caracteriza por tener muy poca experiencia previa docente. Bajo nuestro punto de vista, un profesor/a novel es aquel que cambia repentinamente de rol, pasa de ser estudiante a docente, y se encuentra con un sinfín de situaciones plagadas de tensiones, dilemas y crisis continuas que le hacen aprender de su propia práctica y del trabajo compartido con sus colegas.

\section{Choque con la realidad: problemática del profesorado novel}

La primera vez que un profesor novel entra en un centro educativo y en un aula le invade una sensación de cosquilleo en el estómago y una lluvia de pensamientos diversos en la mente. Veenman (1988) citado por Marcelo (1993) acuña esta situación como "choque de la realidad". Este choque es fácilmente comprensible si tenemos en cuenta como al profesorado poco experimentado se le responsabiliza completamente de un grupo-clase y para enfrentarse a ello tiene que activar estrategias que reduzcan la incertidumbre y la inseguridad que la nueva situación le genera. Además, sufre su primera crisis de identidad profesional, al descubrir la falta de congruencia entre la realidad de la enseñanza y la imagen que cada uno había forjado de ella durante su período de formación inicial.

Desde la década de los 60 se han desarrollado estudios que abordan este tema y concretamente, las problemáticas a las que se enfrenta el profesorado novel. A continuación presentamos dos investigaciones, altamente relacionados con nuestro artículo, y que dan cuenta de las preocupaciones de estos docentes.

En primer lugar describiremos el estudio de Veenman (1984). Este autor llevó a cabo una síntesis de 83 investigaciones realizadas en Estados Unidos y Europa y elaboró un listado con las 24 dificultades a las que tenía que hacer frente el profesorado en su primer año de docencia. Veenman acuñó que la principal preocupación a la que se enfrentaba el profesorado novel era a la disciplina en el aula, seguida de la 
motivación del alumnado y de la adaptación de la enseñanza a las diferencias individuales. En la última posición, se encontraba el número de alumnado por aula. En los resultados de este estudio trataremos de ver si estas problemáticas se reproducen en la actualidad o si bien difieren pasadas más de tres décadas. En este sentido debemos ser muy cautos dado que el contexto de la revisión de Veenman se realizó en Europa y Estados Unidos, y el estudio que aquí se presenta se hace en una zona muy concreta del Estado español, Castellón y Tarragona, con un sistema educativo diferente. Además de ello, el tamaño muestral difiere en gran medida, en nuestra investigación contaremos con 50 docentes. Por este motivo queremos dejar claro que no se realizará un estudio comparativo, sino una aproximación sobre similitudes en cuanto a las problemáticas encontradas por el profesorado novel.

Por otra parte, Marcelo (1991) sigue la base del estudio de Jordell (1985) en el que se identificaban los problemas de enseñanza a los que se tenía que enfrentar el profesorado novel durante su primer año de docencia. Adaptó el Ideario de Problemas de enseñanza de Jordell que constaba de 68 ítems y lo redujo a 55. Cabe destacar cómo en este estudio, al profesorado novel le preocupaba: el tiempo para explicar los contenidos, el elevado número de alumnado en el aula y la escasez de materiales en el centro donde se encuentra.

Lo que sí parece estar claro es que la figura del profesorado novel no está exento de dilemas y en definitiva, de preocupaciones, que o bien pueden convertirse en un reto que le harán mejorar día a día o en el peor de los casos pueden convertirse en una barrera que le impedirán asumir su trabajo como un proceso de aprendizaje continuo.

Revisadas algunas de las conceptualizaciones del profesorado novel, los rasgos más característicos del mismo y sus problemas y preocupaciones, a continuación pasamos a desarrollar cada una de las cuestiones clave que conforman este estudio.

\section{Objetivos y preguntas de investigación}

La finalidad de esta investigación es doble. Por una parte, consiste en conocer, desde la perspectiva de los propios docentes, cuáles son las preocupaciones y los problemas más relevantes a los que se enfrenta el profesorado novel de Educación Secundaria Obligatoria, Bachillerato y Formación Profesional; y por otra, analizar si existen diferencias respecto a las preocupaciones y problemas que posee el profesorado novel, a partir de las opiniones del profesorado de Educación Secundaria Obligatoria, Bachillerato y Formación Profesional dependiendo de sus años de experiencia docente (de 0 a 4 años, de 5 a 10 años y más de 10 años).

Así pues, nuestras preguntas de investigación son las siguientes: ¿Cuáles son las problemáticas más habituales a las que se enfrenta el profesorado de Educación Secundaria Obligatoria, Bachillerato y Formación Profesional? ¿Existen diferencias en estas problemáticas aludidas si tenemos en cuenta los años de experiencia docente? 


\section{Descripción del estudio}

\section{Metodología}

En este trabajo se ha utilizado una metodología cuantitativa basada en el método descriptivo dado que se miden, evalúan y recolectan datos sobre diferentes aspectos, dimensiones o componentes del fenómeno a investigar, en este caso las preocupaciones y problemáticas del profesorado novel (Hernández, Fernández y Baptista, 2001:117). Además, se ha optado por un diseño de encuesta puesto que nos va a permitir recoger información mediante la formulación de preguntas que pretenden realizar estimaciones de las conclusiones a la población de referencia, en este caso al profesorado, a partir de los resultados obtenidos de la muestra.

\section{Participantes}

La población sobre la que se ha realizado la encuesta pertenece a la Comunidad Valenciana y a Cataluña. La muestra está formada por 50 docentes, de los que un 56\% son mujeres y un $44 \%$ son hombres. Por una parte, un $50 \%$ de la muestra es profesorado de Educación Secundaria, un $40 \%$ es profesorado de Formación Profesional, y el resto, un 10\%, es profesorado de Bachillerato. De ellos, un 90\% del profesorado encuestado pertenece a centros públicos, y un $10 \%$ a centros privados. En cuanto a la edad del profesorado, comentar que un $74 \%$ de la muestra son personas con una edad superior a los 30 años, un $16 \%$ tienen entre 25 y 30 años, y un $10 \%$, tienen entre 20 y 25 años. Por su parte, un $46 \%$ de la muestra tiene menos de 4 años de experiencia docente, un $18 \%$ tienen entre 5 y 10 años de experiencia docente, y finalmente, un $36 \%$ de la muestra tiene más de 10 años de experiencia docente. Si cruzamos ambas variables, nivel de estudios impartidos y años de experiencia, la muestra queda representada de la siguiente forma tal y como se aprecia en la tabla 1.

\begin{tabular}{lcccc}
\hline \multirow{2}{*}{$\begin{array}{l}\text { Años de experiencia } \\
\text { docente }\end{array}$} & \multicolumn{3}{c}{ Nivel estudios impartido } & Total \\
\cline { 2 - 5 } & Secundaria & Bachiller & Ciclo Formativo & \\
\hline de 0 a 4 años & 15 & 0 & 8 & 23 \\
\hline de 5 a 10 años & 5 & 0 & 4 & 9 \\
\hline más de 10 años & 7 & 5 & 6 & 18 \\
\hline Total & 27 & 5 & 18 & 50 \\
\hline
\end{tabular}

Tabla 1: Años de experiencia/Nivel de estudios impartido

\section{Instrumento}

Para dar respuesta a las preguntas de investigación planteadas, se ha utilizado el Inventario de Problemas de la enseñanza de Jordell (traducido y adaptado por Marcelo García). Este cuestionario se estructura en 55 ítems, y consiste en una escala tipo Likert, con 5 niveles de respuesta (1: Ningún problema; 2: Algún problema; 3 : Problema considerable; 4: Gran problema; y 5: No relevante para mi nivel). Los ítems hacen referencia a una serie de preocupaciones y problemas a los que ha de hacer frente el profesorado novel, organizadas en las siguientes dimensiones: enseñanza, planificación, evaluación, recursos, entorno, tiempo, relaciones y personal. 


\section{Proceso de análisis de datos}

La encuesta se creó a través de la plataforma Google Drive y se realizó un envío a las direcciones de correo electrónico de los centros de Educación Secundaria y Formación Profesional de la provincia de Castellón y Tarragona en el año 2014. Para el tratamiento estadístico de los datos se ha utilizado el programa SPSS (versión 19 para Windows) al tratarse del software más adecuado para nuestro estudio. Por una parte, se ha llevado a cabo un análisis descriptivo de los resultados obtenidos a través de la encuesta administrada, agrupados en las dimensiones de las que consta la misma, y concretamente se ha realizado un análisis de frecuencias relativas para responder al primero de los objetivos. Por otra parte, se ha utilizado la t de Student, para comprobar si existen diferencias significativas entre los tres grupos y que permitirán responder al segundo de los objetivos

Tal y como podrá apreciarse cuando se presenten los resultados, y siguiendo el trabajo de Marcelo (1991), se considera que son un problema o preocupación aquellos ítems que obtienen un porcentaje superior al 25\%, al sumar las respuestas de los niveles 3 (problema considerable) y 4 (gran problema).

\section{Resultados}

Para hacer más comprensibles los resultados se estructurarán teniendo en cuenta los objetivos planteados en este estudio.

Conocer, desde la perspectiva de los propios docentes, cuáles son las preocupaciones y los problemas más relevantes a los que se enfrenta el profesorado novel de Educación Secundaria Obligatoria, Bachillerato y Formación Profesional

Para abordar el objetivo general de la investigación vamos a dividir la información tomando como referencia las diferentes dimensiones que aparecen en el cuestionario utilizado.

\section{Dimensión académica}

Esta dimensión hace referencia a la metodología, los contenidos y las evaluaciones del estudiantado. Los resultados obtenidos se pueden ver en la siguiente tabla. La última de las columnas $(3+4)$ hace referencia a los ítems que se consideran un problema (recuérdese que han de obtener un porcentaje superior al $25 \%$, al sumar las respuestas de los niveles 3 y 4 ). (Tabla 2 ) 


\begin{tabular}{|c|c|c|c|c|c|c|}
\hline & 1 & 2 & 3 & 4 & 5 & $3+4$ \\
\hline 3. Motivar al alumnado. & $20 \%$ & $48 \%$ & $24 \%$ & $8 \%$ & $0 \%$ & $32 \%$ \\
\hline 4. Explicar las lecciones al alumnado. & $56 \%$ & $42 \%$ & $2 \%$ & $0 \%$ & $0 \%$ & $2 \%$ \\
\hline 5. Introducir nuevas actividades de enseñanza-aprendizaje. & $26 \%$ & $60 \%$ & $14 \%$ & $0 \%$ & $0 \%$ & $14 \%$ \\
\hline $\begin{array}{l}\text { 6. Tratar al alumnado de forma diferenciada e } \\
\text { individualizada. }\end{array}$ & $20 \%$ & $36 \%$ & $26 \%$ & $18 \%$ & $0 \%$ & $44 \%$ \\
\hline 7. Ser creativo/a al enseñar. & $22 \%$ & $50 \%$ & $24 \%$ & $4 \%$ & $0 \%$ & $28 \%$ \\
\hline 9. Conocer aquello que ya sabe el alumnado. & $38 \%$ & $58 \%$ & $4 \%$ & $0 \%$ & $0 \%$ & $4 \%$ \\
\hline 10. Conocer a qué nivel presentar los contenidos. & $32 \%$ & $54 \%$ & $12 \%$ & $2 \%$ & $0 \%$ & $12 \%$ \\
\hline $\begin{array}{l}\text { 11. Saber en qué contenido hacer mayor hincapié o } \\
\text { desglosarlo más. }\end{array}$ & $34 \%$ & $58 \%$ & $8 \%$ & $0 \%$ & $0 \%$ & $8 \%$ \\
\hline 18. Cometer errores de contenido cuando estoy explicando. & $46 \%$ & $46 \%$ & $2 \%$ & $2 \%$ & $4 \%$ & $4 \%$ \\
\hline $\begin{array}{l}\text { 19. No tener suficientes conocimientos de las asignaturas que } \\
\text { enseño. }\end{array}$ & $52 \%$ & $34 \%$ & $14 \%$ & $0 \%$ & $0 \%$ & $14 \%$ \\
\hline 20. Hacer exámenes. & $64 \%$ & $26 \%$ & $6 \%$ & $0 \%$ & $4 \%$ & $6 \%$ \\
\hline 21. Evaluar el nivel de aprendizaje del alumnado. & $44 \%$ & $36 \%$ & $18 \%$ & $0 \%$ & $2 \%$ & $18 \%$ \\
\hline 22. Conocer si mi enseñanza es eficaz. & $24 \%$ & $54 \%$ & $20 \%$ & $2 \%$ & $0 \%$ & $22 \%$ \\
\hline
\end{tabular}

Tabla 2: Frecuencia de las dificultades de la dimensión académica

En esta dimensión se aprecian tres frecuencias superiores al $25 \%$ que se corresponden con el ítem número 6 "Tratar al alumnado de forma diferenciada e individualizada" (44\% de la muestra), el ítem número 3 "Motivar al alumnado" (32\% de la muestra) y el ítem número 7 "Ser creativo/a al enseñar" (28\% de la muestra). Cabe señalar que el ítem con un menor porcentaje de respuestas es el número 4 ( $2 \%$ de la muestra), el cual hace referencia a "Explicar las lecciones al estudiantado", lo cual representa una nula dificultad en la muestra estudiada.

\section{Dimensión de dificultades organizativas}

Esta dimensión se relaciona con la planificación y organización de la enseñanza, y con la distribución del tiempo de trabajo por parte del profesorado. En la tabla 3 se aprecian los diferentes resultados: 


\begin{tabular}{|c|c|c|c|c|c|c|}
\hline & 1 & 2 & 3 & 4 & 5 & $3+4$ \\
\hline 1. Mantener una adecuada organización académica en clase. & $32 \%$ & $58 \%$ & $8 \%$ & $2 \%$ & $0 \%$ & $10 \%$ \\
\hline $\begin{array}{l}\text { 2. Organizar algunas actividades en el aula (por ejemplo: } \\
\text { trabajo en grupo). }\end{array}$ & $26 \%$ & $54 \%$ & $14 \%$ & $6 \%$ & $0 \%$ & $20 \%$ \\
\hline $\begin{array}{l}\text { 12. Estar presionado/a por el tiempo en el que tienen que } \\
\text { cubrir los contenidos. }\end{array}$ & $14 \%$ & $46 \%$ & $18 \%$ & $22 \%$ & $0 \%$ & $40 \%$ \\
\hline 13. Decidir cuánta cantidad de contenido enseñar. & $28 \%$ & $48 \%$ & $22 \%$ & $2 \%$ & $0 \%$ & $24 \%$ \\
\hline 14. Programar una lección. & $40 \%$ & $46 \%$ & $12 \%$ & $2 \%$ & $0 \%$ & $14 \%$ \\
\hline $\begin{array}{l}\text { 15. Programar para un periodo de tiempo más prolongado } \\
\text { (quincena, mes, año). }\end{array}$ & $26 \%$ & $58 \%$ & $14 \%$ & $2 \%$ & $0 \%$ & $16 \%$ \\
\hline 16. Organizar el trabajo diario del aula. & $58 \%$ & $38 \%$ & $4 \%$ & $0 \%$ & $0 \%$ & $4 \%$ \\
\hline $\begin{array}{l}\text { 28. No tener suficiente tiempo libre para dedicar al } \\
\text { alumnado. }\end{array}$ & $22 \%$ & $42 \%$ & $22 \%$ & $10 \%$ & $4 \%$ & $32 \%$ \\
\hline $\begin{array}{l}\text { 40. Tener insuficiente información sobre las normas y } \\
\text { rutinas del centro. }\end{array}$ & $42 \%$ & $48 \%$ & $8 \%$ & $2 \%$ & $0 \%$ & $10 \%$ \\
\hline $\begin{array}{l}\text { 42. Haber encontrado condiciones de trabajo más difíciles } \\
\text { que otros/as profesores/as en el centro (clases más } \\
\text { numerosas, peores, mayor carga de trabajo). }\end{array}$ & $38 \%$ & $46 \%$ & $12 \%$ & $2 \%$ & $2 \%$ & $14 \%$ \\
\hline 45. Elevado número de alumnado en el aula. & $34 \%$ & $34 \%$ & $12 \%$ & $18 \%$ & $2 \%$ & $30 \%$ \\
\hline $\begin{array}{l}\text { 46. Escasez de departamentos y zonas de lectura en el } \\
\text { centro. }\end{array}$ & $34 \%$ & $20 \%$ & $32 \%$ & $12 \%$ & $2 \%$ & $44 \%$ \\
\hline 47. Encontrar tiempo para preparar los materiales. & $24 \%$ & $42 \%$ & $28 \%$ & $6 \%$ & $0 \%$ & $34 \%$ \\
\hline $\begin{array}{l}\text { 48. Encontrar tiempo para leer libros y revistas } \\
\text { profesionales. }\end{array}$ & $22 \%$ & $38 \%$ & $28 \%$ & $10 \%$ & $2 \%$ & $38 \%$ \\
\hline $\begin{array}{l}\text { 51. Distancia del centro en referencia a mi domicilio } \\
\text { particular. }\end{array}$ & $56 \%$ & $14 \%$ & $16 \%$ & $10 \%$ & $4 \%$ & $20 \%$ \\
\hline
\end{tabular}

Tabla 3: Frecuencia de las dificultades organizativas

En esta dimensión podemos observar que se encuentran 6 frecuencias superiores al 25\%, siendo el más alto el ítem número 46 "Escasez de departamentos y zonas de lectura en el centro" (44\% de la muestra), seguido del ítem número 12 "Estar presionado/a por el tiempo en el que tienen que cubrir los contenidos" ( $40 \%$ de la muestra), del ítem número 48 "Encontrar tiempo para leer libros y revistas profesionales" (38\% de la muestra), del ítem 47 "Encontrar tiempo para preparar los materiales" (34\% de la muestra), del ítem 28 "No tener suficiente tiempo libre para dedicar al alumnado" (32\% de la muestra) y finalmente, del ítem 45 "Elevado número de alumnado en el aula" (30\% de la muestra).

Por el contrario el ítem perteneciente a esta dimensión que menos preocupa al profesorado es el número 16 “Organizar el trabajo diario del aula” (4\% de la muestra).

\section{Dimensión de dificultades sociales}

En esta dimensión se engloban los ítems relacionados con las interacciones que el docente mantiene con el alumnado, los aspectos que pueden ser conflictivos en el trato $\mathrm{y}$ las relaciones con los padres y madres, el ambiente y las relaciones entre 
compañeros/as del centro y la dirección. En la tabla número 4 se pueden observar los resultados de esta dimensión:

\begin{tabular}{|c|c|c|c|c|c|c|}
\hline & 1 & 2 & 3 & 4 & 5 & $3+4$ \\
\hline 23. Definir mi papel como profesor/a. & $46 \%$ & $50 \%$ & $2 \%$ & $0 \%$ & $2 \%$ & $2 \%$ \\
\hline 24. Saber si gusto al alumnado. & $54 \%$ & $36 \%$ & $2 \%$ & $2 \%$ & $6 \%$ & $4 \%$ \\
\hline 25. Entablar contacto personal con el alumnado. & $66 \%$ & $22 \%$ & $4 \%$ & $0 \%$ & $8 \%$ & $4 \%$ \\
\hline $\begin{array}{l}\text { 26. Ser más estricto/a con el alumnado que más me } \\
\text { gusta. }\end{array}$ & $24 \%$ & $54 \%$ & $16 \%$ & $2 \%$ & $4 \%$ & $18 \%$ \\
\hline 29. Problemas de disciplina con el alumnado. & $22 \%$ & $56 \%$ & $10 \%$ & $10 \%$ & $2 \%$ & $20 \%$ \\
\hline $\begin{array}{l}\text { 30. No tener suficiente información sobre el alumnado } \\
\text { y su ambiente familiar. }\end{array}$ & $20 \%$ & $56 \%$ & $16 \%$ & $8 \%$ & $0 \%$ & $24 \%$ \\
\hline 31. Relaciones con los padres y madres. & $36 \%$ & $38 \%$ & $2 \%$ & $8 \%$ & $16 \%$ & $10 \%$ \\
\hline 32. Encontrar indiferencia en los padres y madres. & $20 \%$ & $32 \%$ & $16 \%$ & $16 \%$ & $16 \%$ & $32 \%$ \\
\hline $\begin{array}{l}\text { 34. Desacuerdos en las relaciones con los padres y } \\
\text { madres. }\end{array}$ & $32 \%$ & $34 \%$ & $12 \%$ & $6 \%$ & $16 \%$ & $18 \%$ \\
\hline 35. Cooperar con los/as compañeros/as. & $58 \%$ & $38 \%$ & $4 \%$ & $0 \%$ & $0 \%$ & $4 \%$ \\
\hline $\begin{array}{l}\text { 36. Tener la oportunidad/tiempo para hablar con los/as } \\
\text { compañeros/as. }\end{array}$ & $50 \%$ & $42 \%$ & $8 \%$ & $0 \%$ & $0 \%$ & $8 \%$ \\
\hline $\begin{array}{l}\text { 37. Sentirme poco integrado/a profesionalmente en el } \\
\text { centro y entre mis compañeros/as. }\end{array}$ & $72 \%$ & $24 \%$ & $4 \%$ & $0 \%$ & $0 \%$ & $4 \%$ \\
\hline $\begin{array}{l}\text { 38. Desacuerdos profesionales con los/as } \\
\text { compañeros/as. }\end{array}$ & $60 \%$ & $38 \%$ & $1 \%$ & $0 \%$ & $0 \%$ & $1 \%$ \\
\hline $\begin{array}{l}\text { 43. Desacuerdo en las relaciones con la dirección del } \\
\text { centro. }\end{array}$ & $66 \%$ & $24 \%$ & $4 \%$ & $0 \%$ & $6 \%$ & $4 \%$ \\
\hline $\begin{array}{l}\text { 49. Encontrar tiempo para estar con la familia y } \\
\text { amigos/as. }\end{array}$ & $38 \%$ & $38 \%$ & $18 \%$ & $6 \%$ & $0 \%$ & $24 \%$ \\
\hline 50. Mantener mi vida privada apartada del centro. & $64 \%$ & $24 \%$ & $10 \%$ & $0 \%$ & $2 \%$ & $10 \%$ \\
\hline $\begin{array}{l}\text { 52. Entablar relaciones nuevas en el entorno del } \\
\text { centro. }\end{array}$ & $64 \%$ & $28 \%$ & $2 \%$ & $2 \%$ & $4 \%$ & $4 \%$ \\
\hline $\begin{array}{l}\text { 53. Limitaciones de la localidad de trabajo respecto a } \\
\text { actividades culturales, servicios, comunicaciones. }\end{array}$ & $56 \%$ & $30 \%$ & $6 \%$ & $6 \%$ & $2 \%$ & $12 \%$ \\
\hline $\begin{array}{l}\text { 54. Calidad del alojamiento fuera de su domicilio } \\
\text { habitual. }\end{array}$ & $54 \%$ & $18 \%$ & $0 \%$ & $6 \%$ & $22 \%$ & $6 \%$ \\
\hline 55. Estar preocupado/a con la enseñanza diaria. & $38 \%$ & $48 \%$ & $14 \%$ & $0 \%$ & $0 \%$ & $14 \%$ \\
\hline
\end{tabular}

Tabla 4: Frecuencia de la dimensión Dificultades sociales.

En este caso podemos ver que únicamente un ítem tiene una puntuación superior al $25 \%$, en este caso es el número 32 "Encontrar indiferencia en los padres y madres" (32\% de la muestra). El ítem que menos preocupa a los docentes, es el número 38 "Desacuerdos profesionales con los/as compañeros/as" (1\% de la muestra), seguido del número 23 "Definir mi papel como profesor/a" (2\% de la muestra).

\section{Dimensión de dificultades materiales-tecnológicas}


Esta dimensión hace referencia a los medios y recursos disponibles que hay en los centros. En la siguiente tabla se pueden apreciar las diferentes frecuencias encontradas en cada uno de los ítems:

\begin{tabular}{|c|c|c|c|c|c|c|}
\hline & 1 & 2 & 3 & 4 & 5 & $3+4$ \\
\hline 8. Escoger el libro de texto. & $28 \%$ & $40 \%$ & $10 \%$ & $6 \%$ & $16 \%$ & $16 \%$ \\
\hline 17. Utilización de medios de enseñanza & $56 \%$ & $34 \%$ & $6 \%$ & $2 \%$ & $2 \%$ & $8 \%$ \\
\hline $\begin{array}{l}\text { 27. Encontrar rechazo por parte del alumnado cuando llevo } \\
\text { a cabo métodos de enseñanza que no están acostumbrados } \\
\text { a utilizar. }\end{array}$ & $24 \%$ & $54 \%$ & $18 \%$ & $0 \%$ & $4 \%$ & $18 \%$ \\
\hline $\begin{array}{l}\text { 33. Encontrar resistencias o escepticismo en los } \\
\text { padres/madres cuando se intenta llevar a cabo nuevos } \\
\text { métodos de enseñanza. }\end{array}$ & $34 \%$ & $26 \%$ & $20 \%$ & $4 \%$ & $16 \%$ & $24 \%$ \\
\hline $\begin{array}{l}\text { 39. Tener información insuficiente sobre como localizar } \\
\text { materiales didácticos. }\end{array}$ & $42 \%$ & $48 \%$ & $8 \%$ & $2 \%$ & $0 \%$ & $10 \%$ \\
\hline $\begin{array}{l}\text { 41. Despertar escepticismo o resistencia en los/as } \\
\text { compañeros/as o la dirección del centro cuando intento } \\
\text { desarrollar nuevos métodos de enseñanza. }\end{array}$ & $58 \%$ & $36 \%$ & $2 \%$ & $0 \%$ & $4 \%$ & $2 \%$ \\
\hline 44. Escasez de materiales didácticos en el centro. & $34 \%$ & $42 \%$ & $10 \%$ & $12 \%$ & $2 \%$ & $22 \%$ \\
\hline
\end{tabular}

Tabla 5: Frecuencia de las dificultades materiales-tecnológicas.

En esta dimensión se observa que no hay ningún ítem que supera el 25\%. El ítem más alto es el número 44 "Escasez de materiales didácticos en el centro" (22\% de la muestra). El resto de ítems han obtenido puntuaciones mayores en las opciones 1 y 2, lo que significa que no les ha supuesto ni grandes ni considerables dificultades. Parece ser que esta dimensión no les resultó o les ha resultado problemática.

Comprobar si existen diferencias respecto a las preocupaciones y problemas que posee el profesorado novel, a partir de las opiniones del profesorado de Educación Secundaria Obligatoria, Bachillerato y Formación Profesional dependiendo de sus años de experiencia docente (de 0 a 4 años, de 5 a 10 años y más de 10 años).

En este apartado presentaremos, por una parte, las medias globales de todas las dimensiones diferenciadas por grupos, por otra, las dos principales preocupaciones percibidas, y finalmente, los ítems que son estadísticamente significativos cuando se realizan las comparativas entre grupos (se han realizado las comparaciones teniendo cuenta todas las combinaciones posibles: grupo de 0 a 4 años con grupo de 5 a 10 años; grupo de 0 a 4 con grupo de más de 10 años y grupo de 5 a 10 años con grupo de más de 10 años).

En la tabla 6 se observa cómo, de forma global, a mayor experiencia docente menos problemáticas se perciben las diferentes dimensiones, encontrándose mayores semejanzas en las puntuaciones en el primer y en el segundo grupo. 


\begin{tabular}{lcccc}
\hline & Dimensión 1 & Dimensión 2 & Dimensión 3 & Dimensión 4 \\
\hline 0 a 4 años & 1,92 & 2.04 & 1,99 & 2.15 \\
\hline 5 a 10 años & 1,94 & 2.17 & 1.85 & 2.17 \\
\hline $\begin{array}{l}\text { más de } 10 \\
\text { años }\end{array}$ & 1,68 & 1.83 & 1.62 & 1.65 \\
\hline
\end{tabular}

Tabla 6: Medias por dimensiones y por grupos

En cuanto a las 3 principales preocupaciones por grupo en la tabla 7 quedan reflejadas todas ellas, encontramos con en el primer y segundo grupo se focalizan en las familias y en el tercero en el estudiantado:

\begin{tabular}{lcc}
\hline \multicolumn{1}{c}{ Grupo } & Principales preocupaciones \\
\hline 0 a 4 años & Ítem 32 Encontrar indiferencia en los padres (3,04) \\
& Ítem 31. Relaciones con los padres (2.78) \\
& Ítem 12. Estar presionado/a por el tiempo en que se han de cubrir los objetivos (2.74) \\
\hline 5 a 10 años & Ítem 33-Encontrar resistencias o escepticismo en los padres cuando se intenta llevar a \\
& & cabo nuevos métodos de enseñanza (3.22) \\
& & Ítem 34-Desacuerdo en las relaciones con los padres (3.11) \\
& & Ítem 32- Encontrar indiferencia en los padres (3.00) \\
\hline $\begin{array}{l}\text { más de } \\
\text { años }\end{array}$ & 10 & Ítem 6- Tratar al alumnado de forma diferenciada e individualizada (2.50) \\
& & Ítem 28- No tener suficiente tiempo libre para dedicar al estudiantado (2.39) \\
& Ítem 45- Elevado número de estudiantes en el aula (2.28) \\
\hline
\end{tabular}

Tabla 7: Principales preocupaciones y medias

Como puede observarse en la siguiente tabla a mayor experiencia docente menos problemáticas se experimentan las situaciones que presentan cada uno de los ítems. Véase en negrita cómo únicamente se da el caso contrario en tres de las cuestiones.

\begin{tabular}{|c|c|c|c|c|}
\hline \multicolumn{5}{|c|}{ DIMENSIÓN 1} \\
\hline \multicolumn{5}{|c|}{ Ítem 20- Hacer exámenes } \\
\hline Grupo & $\mathbf{N}$ & Media & $\begin{array}{c}\text { Desviación } \\
\text { típica }\end{array}$ & t de Student \\
\hline 0 a 4 años & 23 & 1,91 & 1,164 & \multirow[t]{2}{*}{.021} \\
\hline 5 a 10 años & 9 & 1,22 & ,441 & \\
\hline \multicolumn{5}{|c|}{ Ítem 11- Saber en qué contenido hacer más hincapié } \\
\hline 0 a 4 años & 23 & 1,87 & ,626 & \multirow[t]{2}{*}{.045} \\
\hline más de 10 años & 18 & 1,50 &, 514 & \\
\hline \multicolumn{5}{|c|}{ Ítem 20- Hacer exámenes } \\
\hline 0 a 4 años & 23 & 1,91 & 1,164 & \multirow{2}{*}{.017} \\
\hline más de 10 años & 18 & 1,22 & ,548 & \\
\hline \multicolumn{5}{|c|}{ Ítem 21- Evaluar el nivel de aprendizaje del alumnado } \\
\hline 0 a 4 años & 23 & 2,17 & ,937 & \multirow[t]{2}{*}{.007} \\
\hline más de 10 años & 18 & 1,44 & ,705 & \\
\hline \multicolumn{5}{|c|}{ Ítem 5- Introducir nuevas actividades de enseñanza-aprendizaje } \\
\hline 5 a 10 años & 9 & 2,22 & 0,441 & \multirow{2}{*}{.035} \\
\hline más de 10 años & 18 & 1,78 & 0,548 & \\
\hline
\end{tabular}




\begin{tabular}{|c|c|c|c|c|}
\hline \multicolumn{5}{|c|}{ DIMENSIÓN 2} \\
\hline \multicolumn{5}{|c|}{ Ítem 51 Distancia centro domicilio } \\
\hline 0 a 4 años & 23 & 1,70 & 1,063 & \multirow[t]{2}{*}{.004} \\
\hline 5 a 10 años & 9 & 3,33 & 1,225 & \\
\hline \multicolumn{5}{|c|}{ Ítem 12 Presionado contenidos } \\
\hline 0 a 4 años & 23 & 2,74 & 1,095 & \multirow[t]{2}{*}{.027} \\
\hline más de 10 años & 18 & 2,06 &, 082 & \\
\hline \multicolumn{5}{|c|}{ Ítem 40 Información normas y rutinas } \\
\hline 0 a 4 años & 22 & 1,68 & ,716 & \multirow[t]{2}{*}{.017} \\
\hline más de 10 años & 18 & 1,22 & 428 & \\
\hline \multicolumn{5}{|c|}{ Ítem 48 Leer revistas profesionales } \\
\hline 5 a 10 años & 9 & 2,67 & ,500 & \multirow[t]{2}{*}{.021} \\
\hline más de 10 años & 18 & 2,00 & 907 & \\
\hline \multicolumn{5}{|c|}{ Ítem 51 Distancia centro } \\
\hline 5 a 10 años & 9 & 3,33 & 1,225 & \multirow[t]{2}{*}{.002} \\
\hline más de 10 años & 18 & 1,50 & 924 & \\
\hline \multicolumn{5}{|c|}{ DIMENSIÓN 3} \\
\hline \multicolumn{5}{|c|}{ Ítem 25 Contacto personal alumnado } \\
\hline 0 a 4 años & 23 & 1,87 & 1,359 & \multirow[t]{2}{*}{.006} \\
\hline 5 a 10 años & 9 & 1,00 &, 000 & \\
\hline \multicolumn{5}{|c|}{ İtem 37 Poco integrado } \\
\hline 0 a 4 años & 23 & 1,48 & ,593 & \multirow[t]{2}{*}{.001} \\
\hline 5 a 10 años & 9 & 1,00 & 000 & \\
\hline \multicolumn{5}{|c|}{ Ítem 38 Desacuerdos profesionales } \\
\hline 0 a 4 años & 23 & 1,22 & ,422 & \multirow[t]{2}{*}{.033} \\
\hline 5 a 10 años & 9 & 1,67 & ,500 & \\
\hline \multicolumn{5}{|c|}{ Ítem 31 Relaciones padres } \\
\hline 0 a 4 años & 23 & 2,78 & 1,622 & \multirow[t]{2}{*}{.002} \\
\hline más de 10 años & 18 & 1,50 & ,618 & \\
\hline \multicolumn{5}{|c|}{ Ítem 34 Desacuerdos padres } \\
\hline 0 a 4 años & 23 & 2,70 & 1,636 & .010 \\
\hline más de 10 años & 18 & 1,67 & ,686 & \\
\hline & & elacior & & \\
\hline 0 a 4 años & 23 & 1,87 & 1,254 & .028 \\
\hline más de 10 años & 18 & 1,22 & ,428 & \\
\hline & Íten & cto per: & & \\
\hline 5 a 10 años & 9 & 1,00 & 000 & .023 \\
\hline más de 10 años & 18 & 1,61 & 1,037 & \\
\hline & & esacue & & \\
\hline 5 a 10 años & 9 & 3,11 & 1,364 & .013 \\
\hline más de 10 años & 18 & 1,67 & ,686 & \\
\hline & & IENSI & & \\
\hline & & Escog & & \\
\hline 0 a 4 años & 23 & 2,78 & 1.650 & .025 \\
\hline más de 10 años & 18 & 1,83 & ,924 & \\
\hline & & scasez & & \\
\hline 0 a 4 años & 23 & 2,39 & 1,270 & .046 \\
\hline más de 10 años & 18 & 1,78 & ,548 & \\
\hline & & lazo $\mathrm{m}$ & & \\
\hline 5 a 10 años & 9 & 2,22 & ,441 & .047 \\
\hline más de 10 años & 18 & 1,78 & 647 & \\
\hline
\end{tabular}




\begin{tabular}{l|c|c|c|c}
\hline \multicolumn{5}{c}{ Ítem 33 Resistencia nuevos métodos } \\
\hline 5 a 10 años & 9 & 3,22 & 1,563 & \multirow{2}{*}{.044} \\
\cline { 1 - 4 } más de 10 años & 18 & 1,94 &, 802 & \\
\hline \multicolumn{5}{c}{ Ítem 41 Escepticismo profesorado nuevos métodos } \\
\hline a 10 años & 9 & 1,78 &, 441 & .077 \\
\hline más de 10 años & 18 & 1,22 &, 428 & \\
\hline
\end{tabular}

Tabla 8: Diferencias entre grupos (N, media, desviación típica, t Student).

\section{Discusión de los resultados}

Respondiendo al objetivo general de esta investigación, podemos ver que las preocupaciones y problemas a los que se enfrenta el profesorado novel de este estudio tienen que ver con cuestiones relacionadas con las dimensiones académicas y organizativas. A esta tipología de docentes les resultan problemáticas dos aspectos básicos. Por un parte, el tratar al estudiantado de forma diferenciada e individualizada, lo cual se encuentra estrechamente relacionado con el elevado número de estudiantes en las aulas y el poco tiempo que tienen para dedicarles. Valores similares se obtienen en el trabajo de Eirín, García y Montero (2007) en que los ítems relacionados con la atención a la diversidad son los que presentan mayor preocupación. Y por otra parte, la falta de motivación del estudiantado. Esta actitud puede deberse a otras preocupaciones que también señalan, como por ejemplo, la poca creatividad que poseen a la hora de enseñar, el no tener tiempo a preparar materiales y realizar lecturas pedagógicas, y a la gran presión a la que se ven sometidos por cubrir los contenidos, ejercida en la mayoría de los casos por los libros de texto. Del mismo modo, en el estudio de Azcárate y Cuesta (2005) una característica compartida por el colectivo era la utilización del libro de texto como el referente fundamental para la selección y la organización de los contenidos. Esta situación daba lugar a tener poco margen de decisión por parte del profesorado, la cual se reducía a la supresión o a la ampliación de las temáticas que el libro de texto ofrecía, atendiendo sobre todo a factores como la falta de tiempo o a la presión de las exigencias de cara a los cursos posteriores.

Aunque paradójicamente no supone ninguna problemática para la muestra de nuestro estudio la dimensión que hace referencia a los materiales y a la tecnología. Estos resultados coinciden parcialmente con los de Cardona (2008) quien encontró que la problemática mayor a las que tenían que hacer frente los profesores/as noveles era el trabajo burocrático, seguido de cómo motivar al alumnado y de la adaptación de la enseñanza a las diferencias individuales.

En la actual sociedad de la información, el estudiantado dispone de infinitas fuentes de información (Naisbitt, 1983; Burstein, Oakes y Guiton, 1992). El profesorado de hoy en día, si quiere adaptarse a esta realidad tiene que buscar alternativas y estar a la última en propuestas metodológicas más activas y participativas, y en las nuevas tecnologías. Además de adoptar mecanismos para la individualización de la enseñanza en los que se tengan en cuenta los estilos de aprendizaje, las capacidades y la diversidad del estudiantado, partiendo de la base de que cada ser humano es irrepetible y único. 
Pero, desafortunadamente, como tan acertadamente nos señala Esteve (2003) existe un problema de velocidades: la rapidez con la que se suceden los cambios sociales es mayor que la capacidad de adaptación y de respuesta de nuestros sistemas educativos. Cuando se han adoptado y concretado medidas para hacer frente a esta realidad esta ha vuelto a cambiar. Esto no debe llevarnos a la desesperación, sino a la concepción de la educación como un reto, como un proceso cambiante y de mejora continua donde el docente ha de estar en continua formación y aprendizaje.

Respecto a la diferenciación que hemos realizado en este estudio teniendo en cuenta los años de experiencia, vemos como a mayor experiencia docente menos problemáticas se perciben las situaciones. Al profesorado novel le preocupa altamente cubrir los objetivos, cuestión que, con el tiempo, va difuminándose. Es coincidente con el profesorado de 5 a 10 años en la problemática relacionada con las familias. A los dos grupos les preocupan las relaciones con los padres y madres y sus resistencias frente a nuevas propuestas de trabajo. En este sentido, se hace necesario plantear propuestas para incluir a las familias en los espacios de enseñanza-aprendizaje. En las conclusiones ofrecemos algunas pinceladas de los mismos. Por su parte, las preocupaciones del profesorado más experimentado se centran alrededor del estudiantado, de las dificultades de realizar una enseñanza individualizada dado el poco tiempo del que disponen para atenderlo y de la ratio tan numerosa de las aulas.

Finalmente, creemos interesante contrastar estos datos en comparación con los dos autores revisados en la introducción teórica. En cuanto a la diferenciación que encontramos en los estudios de Veenman (1984) y Marcelo (1991), se observa como en el caso del primer autor, aparece en primera posición, y nuevamente, la presión por el tiempo en el que se deben cubrir los objetivos, en cambio Veenman plantea la disciplina en el aula como una de sus mayores preocupaciones. Por lo que se presume, en la actualidad y con el profesorado de nuestro estudio, no existen tantas problemáticas a la hora de gestionar el aula sino que hay una mayor preocupación en dar a tiempo todos los contenidos programados desde los departamentos. Otro de los datos relevantes que aparecen en el trabajo de Veenman (1984) es la poca problemática que supone el número de estudiantes en el aula. Tres décadas más tarde vemos como esta situación se destaca como una de las mayores preocupaciones de los docentes. Con las nuevas reformas educativas esta situación se recrudece y vemos como es uno de los temas clave de muchas de las movilizaciones que se están sucediendo en nuestro país.

Con el estudio de Marcelo (1991) podemos constatar que, por mucho que su investigación se realizó hace 23 años, se encuentran muchas similitudes. Para comenzar, en la primera posición, se sitúa en ambos estudios el mismo ítem: la presión por el tiempo en el que se han de cubrir los contenidos. Por su parte, de la segunda posición hasta la octava, cabe destacar que se encuentran todos los ítems pero ordenados con diferente posición, a excepción del ítem número 7 "Ser creativo" que en nuestra muestra se encuentra en cuarta posición y en el estudio de Marcelo se sitúa en penúltima posición. Por tanto, podemos decir que, a pesar de que la sociedad ha cambiado, las preocupaciones del profesorado, en mayor o menor medida son las mismas que hace más de dos décadas. 


\section{Conclusiones}

En esta investigación ha quedado patente cómo una de las mayores preocupaciones del profesorado novel en la actualidad es acabar el temario y abordar todos los contenidos de las asignaturas. Tal y como se indica en el Informe Delors (1996) no se trata solo de aprender o de conocer, este aprendizaje conceptual debe complementarse con el aprender a hacer, y el aprender a ser, a vivir juntos. Tradicionalmente se ha perseguido el aprendizaje memorístico y el almacenamiento de la información, pero tal y como nos señala Gardner y Boix (1994: 203, citado en Marcelo, 2001): menos es más. Parece ser que, el principal enemigo de la comprensión es completar el temario. Por tanto, desde nuestro punto de vista hay dos cuestiones fundamentales a trabajar con el profesorado novel. Por una parte, el adoptar estrategias y metodologías didácticas que permitan al estudiantado aprender haciendo, como el Aprendizaje-Servicio, aprender enseñando, como la Tutoría entre Iguales, o bien aprender compartiendo, como el Aprendizaje Cooperativo. Y por otra, mecanismos de colaboración entre el profesorado y entre el profesorado y las familias. El aislamiento de los docentes está favorecido por la arquitectura escolar, que organiza los centros en módulos estándar, así como por la distribución del tiempo y el espacio, y la existencia de normas de independencia y privacidad entre los profesores (Marcelo, 2011). A pesar de ello, la educación del siglo XXI exige del trabajo colectivo de docentes y familias, y de espacios que permitan al profesorado formarse y compartir sus preocupaciones, experiencias y vivencias en las aulas y en los centros. En este sentido, es el profesorado con menos experiencia el que más se beneficiará de estas estrategias que le permitirán por una parte, conocer el trabajo de otros/as compañeros/as, y por otra, compartir los miedos y preocupaciones que, seguramente, ya han vivido la mayoría de sus iguales. Por este motivo, consideramos relevante mencionar algunas propuestas e iniciativas que ayudarían a mitigar muchos de los miedos y preocupaciones que afectan al profesorado novel, como son los Grupos de Apoyo entre Profesores/as, el Estudio de clases y el Mentoring. La primera de ellas, parte de la base de que los profesores tienen conocimientos y destrezas que pueden ser compartidos con sus compañeros/as. Proporcionan una oportunidad para que el profesorado de un mismo centro comparta, analice y resuelva conjuntamente sus problemas (Gallego, 1998). En cuanto al Estudio de Clases es un proceso mediante el cual los profesores trabajan en común para mejorar progresivamente sus métodos pedagógicos, examinándose y criticándose mutuamente las técnicas de enseñanza (Moliner y Loren, 2010). Finalmente, el Mentoring hace referencia a la relación que se establece entre una persona con menos experiencia y un/a compañero/a más experimentado que le guía y apoya en diferentes contextos (Falchikov, 2001).

Como puede observarse todas estas propuestas tienen un denominador común, la colaboración. Esta se concibe como un proceso relacional en el que participan determinados profesionales dotados de un cierto bagaje de conocimientos, capacidades y habilidades. A partir de ellos, se contribuye a configurar contextos de trabajo, en colaboración, para la utilización adecuada del conocimiento disponible en la resolución de problemas que tienen que ver con la práctica educativa y su mejora (Nieto, 1996). 
Nos gustaría finalizar este artículo anotando algunas de las limitaciones encontradas en este trabajo. En este estudio nos hemos centrado fundamentalmente en las problemáticas y las preocupaciones del profesorado novel de una zona en concreto, la provincia de Castellón y la de Tarragona. Una de las propuestas de mejora sería ampliar dicha muestra tanto en número de provincias como en número de participantes. Además, se podría llevar a cabo un estudio comparativo en profundidad donde se abordaran si existen diferencias significativas entre las opiniones aportadas por el profesorado atendiendo a los rangos de experiencia docente, en este caso en los tres bloques: más de 10 años, de 5 a 10 años y de 0 a 4 años. Del mismo modo, podría ser de gran interés analizar si se encuentran diferencias en las percepciones entre el profesorado que realiza su labor docente en centros públicos y aquel que la desempeña en centros privados. Por otra parte, sería muy interesante el poder contrastar las respuestas obtenidas en los cuestionarios con información de corte cualitativa donde se pudiera profundizar sobre la temática. Los grupos de discusión podrían ser una herramienta muy útil para que el profesorado reflexionara sobre los miedos y las preocupaciones a partir del diálogo y la reflexión entre iguales.

\section{Referencias bibliográficas}

AZCÁRATE, P. Y CUESTA, J. (2005). El profesorado novel de secundaria y su práctica. Estudio de un caso en las áreas de ciencias. Enseñanza de las ciencias, 23(3), 393-402.

BREAUSER, E., DUPONT, P., QUENON, C., Y VILAN, M. (1984). L'enseignant débutant. Bruxelles: Ministére de l'Education Nationale.

BURSTEIN, L., OAKES, J. Y GUITON, G. (1992). Education Indicators, en M.C. Alkin (Ed.), Encyclopedia of Educational Research, 409-418. McMillan: New York.

CAÑÓN, R. (2012). Iniciación a la docencia de los maestros de Educación Primaria (Tesis Doctoral). Universidad de León, León.

CARDONA, J. (2008). Problemática actual del profesorado. Algunas soluciones. Enseñanza, 26, 29- 56.

DELORS, J. (Coord) (1996). La educación encierra un tesoro. Informe a la UNESCO de la Comisión Internacional sobre la educación para el siglo XXI. Madrid: Santillana.

ESTEVE, J.M. (2003). La Tercera Revolución Educativa. La educación en la sociedad del conocimiento Barcelona: Paidós.

EIRÍN, R., GARCÍA, H. M., Y MONTERO, L. (2009). Profesionales principiantes e iniciación profesional. Estudio Exploratorio. Profesorado. Revista de currículum y formación del profesorado, 13(1), 101-115.

FEIMAN-NEMSER, S. (2001). From Preparation to Practice: Designing a Continuum to Strengthen and Sustain Teaching. Teachers College Record, 103(6), 1013-1055. 
GALLEGO, C. (1998). Grupos Colaborativos de Apoyo entre Profesores para la Atención a la Diversidad, en R. Pérez (Coord.), Educación y Diversidad, 513-526. Oviedo: Servicio de Publicaciones de la Universidad de Oviedo.

GARDNER, H., Y BOIX, V. (1994). Teaching for understanding in the disciplines and beyond. Teachers College Record, 96(2), 198-218.

JOHNSTON, J., Y RYAN, K. (1983). Research on the Beginning Teacher: Implications for Teacher Education. En Howey, K. y Gardner, W. (eds.). The Education of Teachers. New York: Longman.

JORDELL, K. (1985). Problems of Begining and More Experiencied teachers in Norway. Scandinavian. Journal of Educational Research, 29 (3) 105-121.

MARCELO, C. (1991). Aprender a enseñar. Un estudio sobre el proceso de socialización de profesores principiantes, Madrid: Ministerio de Educación y Ciencia.

MARCELO, C. (1993). El primer año de enseñanza. Análisis del proceso de socialización de profesores principiantes. Revista do Educación, 300, 225- 277.

MARCELO, C. (1993). Cómo conocen los profesores la materia que enseñan. Algunas contribuciones de la investigación sobre conocimiento didáctico del contenido, en L. Montero y J.M. Vez (Eds.), Las Didácticas Específicas en la Formación del Profesorado (1), 151-186. Santiago de Compostela: Tórculo.

MARCELO, C. (2001). Aprender a enseñar para la sociedad del conocimiento. Revista Complutense de Educación, 12(2), 531-593.

MARCELO, C. (2009). Los comienzos en la docencia: Un profesorado con buenos principios. Profesorado: Revista De Currículum y Formación Del Profesorado, 13(1), 1-25.

MARCELO, C. (2011). Evaluación del desarrollo profesional docente. Coruña: Editorial Davinci.

MOLINER L. Y LOREN C. (2010). La Formación Continua como proceso clave en la profesionalización docente: buenas prácticas en Chile. Revista Latinoamericana de Inclusión Educativa, 4(1), 25-44.

NAISBITT, J. (1983). Macrotendencias: diez nuevas orientaciones que están transformando nuestras vidas. Barcelona: Mitre.

NEMIÑA, R., GARCIA, R., Y MONTERO, L. (2009). Profesores principiantes e iniciación profesional. Estudio exploratorio. Profesorado, Revista de currículum y formación del profesorado, 13(1), 101-115.

NIETO, J. M. (1996). Reconstruir el asesoramiento pedagógico como práctica de apoyo a los centros educativos. Revista de Educación, 311, 217-234.

SAINT-ONGE, M. (1997). Yo explico, pero ellos.... ¿aprenden? Bilbao: Mensajero. 
VEENMAN, S. (1984). Perceived problems of beginning teachers. Review of Education of Research, 54(2), 143-178.

VERA, J. (1988). El profesor principiante. Las dificultades de los profesores en los primeros años de trabajo en la enseñanza. Valencia: Promolibro.

\section{Correspondencia con los autores}

Lidón MOLINER MIRAVET

Universitat Jaume I de Castellón

Departamento de Educación

Avda. Sos Baynat s/n

12071 Castellón

e-mail:mmoliner@uji.es

Joan ORTÍ PITARCH

Universitat Jaume I de Castellón

Departamento de Educación

Avda. Sos Baynat s/n

12071 Castellón

e-mail: al282909@uji.es 